This item was submitted to Loughborough's Research Repository by the author.

Items in Figshare are protected by copyright, with all rights reserved, unless otherwise indicated.

\title{
Embedding OFDM-based carrier communication into power control loop of converter in DC microgrids
}

\section{PLEASE CITE THE PUBLISHED VERSION}

https://doi.org/10.1109/TIE.2021.3095821

\section{PUBLISHER}

Institute of Electrical and Electronics Engineers

\section{VERSION}

AM (Accepted Manuscript)

\section{PUBLISHER STATEMENT}

(c) 2021 IEEE. Personal use of this material is permitted. Permission from IEEE must be obtained for all other uses, in any current or future media, including reprinting/republishing this material for advertising or promotional purposes, creating new collective works, for resale or redistribution to servers or lists, or reuse of any copyrighted component of this work in other works.

\section{LICENCE}

\section{All Rights Reserved}

\section{REPOSITORY RECORD}

Zhang, Ruoqi, Yue Hui, Jiande Wu, Ruichi Wang, Zhengyu Lin, and Xiangning He. 2021. "Embedding Ofdmbased Carrier Communication into Power Control Loop of Converter in DC Microgrids". Loughborough University. https://hdl.handle.net/2134/14883957.v1. 


\title{
Embedding OFDM-Based Carrier Communication into Power Control Loop of Converter in DC Microgrids
}

\author{
Ruoqi Zhang, Student Member, IEEE, Yue Hui, Jiande Wu, Member, IEEE, \\ Ruichi Wang, Member, IEEE, Zhengyu Lin, Senior Member, IEEE, and Xiangning He, Fellow, IEEE
}

\begin{abstract}
In direct current (DC) microgrids, communication between converters is necessary for better power distribution and finer energy scheduling. This paper proposes an orthogonal frequency division multiplexing (OFDM)-based carrier communication method for DC microgrid applications. The method integrates the communication function in the process of power conversion by modulating data into power control loop. To maximize the communication rate in a narrow band limited by PWM control, OFDM technology is applied in the data modulation process. The data is modulated to multiple low-frequency signal carriers, then the modulated wave is added to the output of the power control loop. Since no additional component is needed for sending data, this method has the advantages of low cost and simple implementation. In order to provide theoretical guidance for system design, the DC microgrid applying the proposed method is modeled. Furthermore, important issues of the communication system are discussed, including data frame format and inter-symbol interference (ISI) problem. At last, a 2 kW DC microgrid experimental platform is established and 9.6 kbps communication rate with a transmission distance of $200 \mathrm{~m}$ is achieved, which verifies the feasibility of the proposed method.
\end{abstract}

Index Terms-carrier communication, orthogonal frequency division multiplexing (OFDM), DC microgrid, power control loop, inter-symbol interference (ISI).

\section{INTRODUCTION}

$\mathrm{P}$ OWER electronics technologies have been widely used in industrial and consumer electronics, such as photovoltaics, electric vehicles (EVs), microgrids and many other fields, for several decades. Recently, as the Internet of Things (IoT) grows [1] and the smart grid emerges [2], there is an increasing demand for power electronic equipment to interact with others. Consequently, the power electronic equipment integrated with communication links is a trend for future development.

Manuscript received March 3, 2021; revised June 6, 2021; accepted June 22 , 2021. This work was supported by the National Natural Science Foundation of China (51977189), in part by the U.K. EPSRC Fellowship scheme (EP/S001662/2), and in part by the European Union's Horizon 2020 research and innovation program (898194). (Corresponding author: Jiande Wu.)

R. Zhang, Y. Hui, J. Wu, and X. He are with the College of Electrical Engineering, Zhejiang University, Hangzhou, 310027, China. (e-mail: rockyzhangped@zju.edu.cn; yuehui@zju.edu.cn; eewjd@zju.edu.cn; hxn@ zju.edu.cn).

R. Wang and Z. Lin are with the School of Mechanical, Electrical and Manufacturing Engineering, Loughborough University, Loughborough, LE11 3TU, U.K. (e-mail: R.Wang@lboro.ac.uk; z.lin@ieee.org).
Due to the attractive characteristics such as high efficiency, good compliance with consumer electronics and simple interface with many renewable energy sources, direct current (DC) microgrids will play a much bigger role in future distributed power systems [3]. Conventionally, a hierarchical control structure, which consists of primary, secondary, and tertiary controls, is adopted for microgrid operation [4]. Droop control method is usually employed in the primary control as its simple implementation. However, it suffers from poor voltage regulation and load sharing [3]. To solve this issue, centralized [4] or cooperative distributed control methods [5]-[9] are employed in the secondary control. By providing additional voltage restoration term to the primary control based on voltage and current information of other converters, better voltage regulation and finer power distribution can be achieved. In general, $10 \mathrm{kbps}$ rate and less than $100 \mathrm{~ms}$ communication delay are required by DC microgrid secondary control methods [9].

In order to achieve data communication in DC microgrids, different technologies have been employed, including fieldbus, wireless communication, and power line communication (PLC). Fieldbus technologies, such as $\mathrm{CAN}$ and Profibus, require independent wiring [10], which is costly and complexes the system design. Compared with fieldbus technologies, wireless communication technologies such as WIFI, Zigbee and Bluetooth, have lower maintenance costs [11]-[12]. However, they do not have good real-time control performance and are subject to interference from related frequency bands. PLC technology is another option that does not require independent wiring [13]-[14]. However, additional controllers and signal coupling circuits are required, which increases the cost.

Power electronic converters have the potential of modulating information with power [15], and this feature can be explored for data communication [16]-[19]. In a pulse-width modulation (PWM) based power converter, the frequency and the phase of the PWM carrier are two degrees of freedom, and can be used for data modulation purpose. In [16], a power/signal dual modulation (PSDM) method was proposed. By changing the PWM carrier frequency according to the transmitting data, the frequency shift keying (FSK) data modulation is achieved, and the data can be demodulated from the input/output voltage ripples of power converter. In [17], a novel method was proposed to integrate communication with power conversion by modulating information into the spread spectrum-based PWM carrier. In [18], PSDM technique is applied in LED drives to realize visible-light communication in a cost-effective way. The above methods employ single PWM carrier for the data modulation, and are named as power/signal dual 
modulation with single carrier (PSDM-SC) method in this paper. PSDM-SC can achieve relatively high communication rate, but the data modulation methods are greatly restricted.

Another way to implement PSDM was proposed in [20]-[22] by superimposing a low-frequency signal carrier on the power control loop, and is named as power/signal dual modulation in control loop (PSDM-CL) method in this paper. In [20], disturbances are added into the control loop of the photovoltaic optimizer as the data carrier, and a $2 \mathrm{kbps}$ communication link among the optimizers is established. In [21], a phase-control-based freedom is introduced to the conventional phase-shifted full bridge control loop to realize communication, and the signal intensity can be regulated by the proposed perturbation depth. In [22], a power talk communication strategy is developed for DC microgrids by adding disturbance on voltage reference, which achieves communication speed of 100 to 1000 baud rate. In PSDM-CL methods, power and data modulations use different carriers, therefore many methods can be selected for data modulation.

Compared with other communication technologies, the PSDM method has the advantages of no additional wiring, less communication circuits, and low manufacturing costs.

However, in practical, the data rate and the maximum transmission distance are the major concerns for PSDM methods. For PSDM-SC method, the strength of the transmitted signal is small and cannot be adjusted, so the communication range is limited. On the contrary, PSDM-CL method proposed in [20]-[22] offers controllable transmission signal strength and longer transmission distance. Whereas, the data carrier frequency of PSDM-CL is a fraction of the PWM frequency, so that the bandwidth of the communication signal is relatively small, which might not be fast enough for the demand of secondary control in DC microgrids.

Orthogonal frequency division multiplexing (OFDM) technology has the advantages of high frequency band utilization [23], which has been fully studied in the communication area but not be introduced to the PSDM-CL method yet. To increase data rate in a narrow band, this paper proposes an OFDM-based PSDM-CL method to set up communication links between converters in the secondary control layer of DC microgrids. The data is modulated to multiple low-frequency signal carriers, then the modulated wave is added to the output of the power control loop. By employing OFDM in the data modulation process, the communication rate can be significantly increased and meet the demand of DC microgrid operation.

This paper is organized as follows. In Section II, the fundamentals of PSDM-CL method are put forward. To increase the communication rate, the OFDM-based PSDM-CL method is proposed. In Section III, the model of the DC microgrid employing the proposed method is set up and the signal transmission gain is analyzed in detail. Furthermore, guidance for power system design is provided. In Section IV, important issues of the communication system are discussed. In Section V, the experiments based on a DC microgrid platform are carried out to verify the correctness of the theory and the feasibility of the proposed method. Finally, conclusions and prospects are given in Section VII.

The main contributions of the paper are as follows.

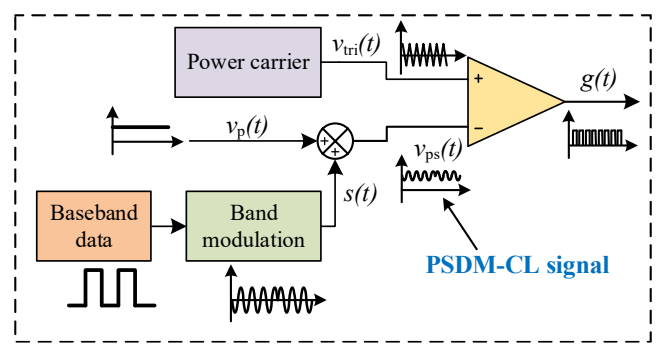

Fig. 1. Data modulation process in a DSC.

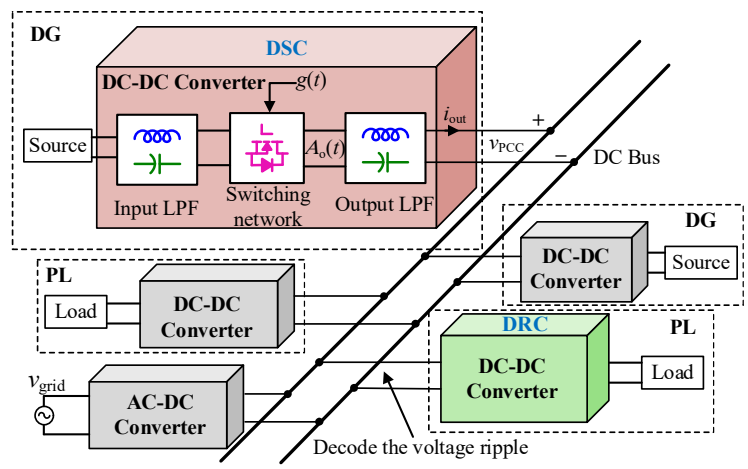

Fig. 2. Simplified structure of a DC microgrid system.

1) For the first time, the OFDM technology is implemented in the PSDM-CL method. A $9.6 \mathrm{kbps}$ communication link is achieved in a $2 \mathrm{~kW}$ DC microgrid system with $10 \mathrm{kHz}$ narrow bandwidth, which is significantly faster than the previous PSDM-CL methods in [20]-[22].

2) A new model of DC microgrid employing the proposed OFDM-based PSDM-CL method is established. The signal transmission gain is analyzed in detail, which offers theoretical guidance for system design.

3) To deal with different and complex communication channel characteristics in the power electronics systems embedding the proposed method, a practical inter-symbol interference (ISI) evaluation approach by numerical computation is designed in this paper.

\section{PRINCIPLE OF OFDM-BASEd PSDM-CL METHOD}

\section{A. Principle of PSDM-CL Method}

In the proposed PSDM-CL system, the power converter sending out data symbols is named as data-sending converter (DSC), while the power converter receiving data is named as data-receiving converter (DRC). The data modulation process in a DSC is shown in Fig. 1. First, the baseband data is modulated as a perturbance signal $s(t)$. In this procedure, conventional modulation methods, such as FSK, amplitude shift keying (ASK), phase shift keying (PSK), and methods of multi-carrier modulation, can be employed. Then, $s(t)$ is added to the output of power control loop $v_{\mathrm{p}}(t)$ as a PSDM-CL signal $v_{\mathrm{ps}}(t)$. At last, $v_{\mathrm{ps}}(t)$ is compared with the triangular carrier $v_{\mathrm{tri}}(t)$ to generate a gate drive signal $g(t)$ for the DSC. After these, the data is modulated and merged into $g(t)$.

In a DC microgrid system, PSDM-CL technique can be adopted by the converters for communication. A simplified structure of DC microgrid is shown in Fig. 2. In the system, any converters, including distributed generators (DGs) and power loads (PLs), can operate as a DSC or a DRC. 


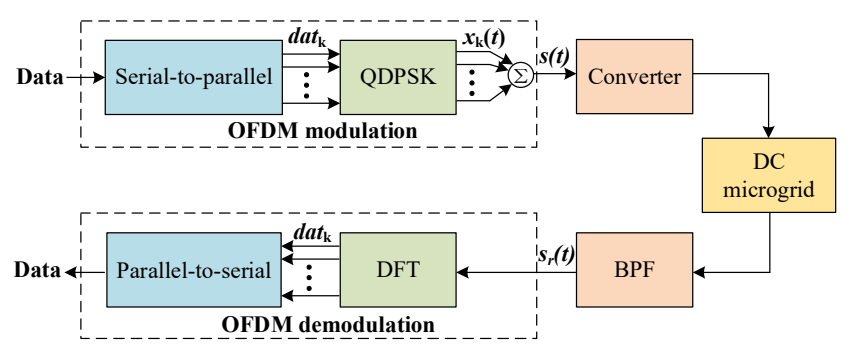

Fig. 3. Communication process of OFDM-based PSDM-CL method.

Generally, a DC-DC converter can be divided into three parts: input low pass filter (LPF), switching network and output LPF. In a DSC, the gate drive signal $g(t)$, which contains data information, is amplified by the switching network as the power pulse $A_{\mathrm{o}}(t)$, which is then filtered by the output LPF. Accompanied with electric power, the data symbol is injected into the DC bus. In a DRC, the DC bus voltage is sampled, and the data can be demodulated.

In PSDM-CL scheme, it is preferable to decouple the control of power transfer and data communication. Thus, in order to suppress the influence of power control on the data symbol, the data carrier frequency $f_{\mathrm{c}}$ should be selected above the power control loop cut-off frequency $f_{\mathrm{p}}$. On the other side, mixed in the output power pulse $A_{0}(t)$, the data symbol is attenuated by the output LPF, so $f_{\mathrm{c}}$ should be chosen as low as possible for higher transmission gain. Considering the aforementioned factors, the data carrier frequency $f_{\mathrm{c}}$ is selected as $1 / 50 \sim 1 / 5$ of the converter's switching frequency $f_{\mathrm{s}}$.

According to above analysis, it can be concluded that the data bandwidth in PSDM-CL scheme is limited in a narrow band. In order to promote the bandwidth efficiency of the communication system, OFDM technique with multiple subcarriers is employed in this paper. For each subcarrier, quadrature differential phase shift keying (QDPSK) is selected as the band modulation method. The principles of data modulation and demodulation are discussed as follows.

B. Data Modulation and Demodulation Principle of PSDM-CL Method Based on QDPSK-OFDM

For an OFDM system with $N$ subcarriers $x_{\mathrm{k}}(t)$, the modulated signal $s(t)$ of the system can be expressed as

$$
\begin{gathered}
s(t)=\sum_{k=1}^{N} x_{\mathrm{k}}(t), \\
x_{\mathrm{k}}(t)=d_{\mathrm{k}} \cos \left(2 \pi f_{\mathrm{ck}} t+\varphi_{\mathrm{k}}\right), k=1,2, \ldots, N,
\end{gathered}
$$

where $x_{\mathrm{k}}(t)$ is the $k^{\text {th }}$ subcarrier, and $d_{\mathrm{k}}, f_{\mathrm{ck}}, \varphi_{\mathrm{k}}$ are the amplitude, frequency, and phase of the $k^{\text {th }}$ subcarrier respectively.

According to the basis of OFDM, $x_{\mathrm{k}}(t)$ should be orthogonal with each other in a single symbol period $T_{\mathrm{b}}$, which is

$$
\int_{t}^{t+T_{\mathrm{b}}} \cos \left(2 \pi f_{\mathrm{ci}} t+\varphi_{\mathrm{i}}\right) \cos \left(2 \pi f_{\mathrm{cj}} t+\varphi_{\mathrm{j}}\right) d t=0,
$$

where $i, j=1,2, \ldots, N$, and $i \neq j$. It can be deduced from (3) that $f_{\text {ck }}$ satisfies

$$
f_{\mathrm{ck}}=\frac{M_{\mathrm{k}}}{T_{\mathrm{b}}}
$$

where $M_{\mathrm{k}}$ are positive integers.

The process of QDPSK-OFDM includes two steps, as shown in Fig. 3. The first step is the serial-to-parallel conversion. The baseband data stream in series is redistributed to $N$ OFDM

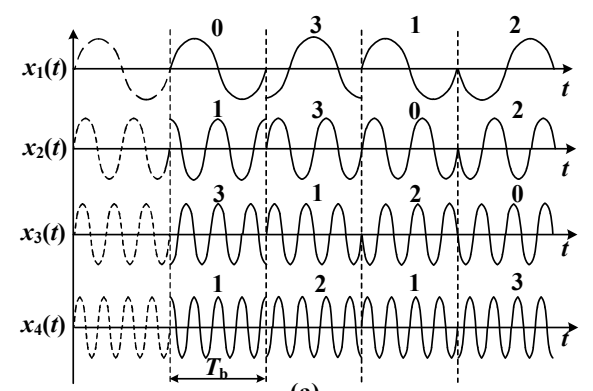

(a)

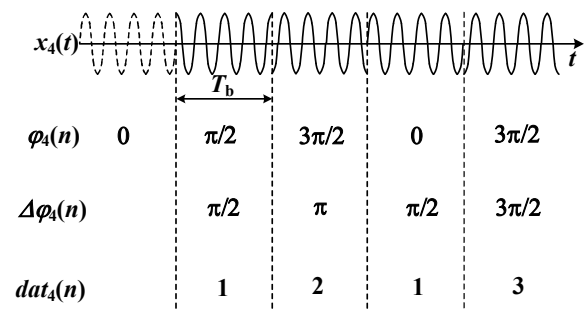

(b)

Fig. 4(a). Modulated subcarrier waveforms of QDPSK-OFDM (b) Details of $x_{4}(t)$.

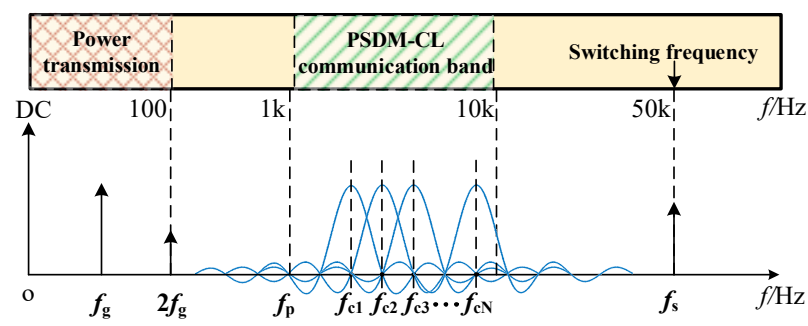

Fig. 5. Bus voltage spectrum of OFDM-based PSDM-CL converter.

subcarriers. The second step is the QDPSK modulation. For each subcarrier $x_{\mathrm{k}}(t)$, a quadrature bit is converted into $M_{\mathrm{k}}$ continuous sinusoidal waves in one symbol period. As the data information of the $k^{\text {th }}$ subcarrier in the $n^{\text {th }}$ symbol period, dat $[n]$ determines the phase difference $\Delta \varphi_{\mathrm{k}}(n)$ of $x_{\mathrm{k}}(t)$ between the $n^{\text {th }}$ and $(n-1)^{\text {th }}$ symbol period, which is

$$
\Delta \varphi_{\mathrm{k}}(n)=\varphi_{\mathrm{k}}(n)-\varphi_{\mathrm{k}}(n-1)=\frac{\pi}{2} \operatorname{dat}_{\mathrm{k}}[n], \operatorname{dat}_{\mathrm{k}}[n] \in\{0,1,2,3\} .
$$

The perturbance signal $s(t)$ is the summation result of all $x_{\mathrm{k}}(t)$, which is then added to the power control loop for transmission.

Suppose a 16 Quaternary code "0312 130231201213 " is being transmitted in an OFDM system with 4 subcarriers. The waveform of $x_{\mathrm{k}}(t)$ is illustrated in Fig. 4(a). Take the fourth subcarrier $x_{4}(t)$ as an example, the transmitted data is "1213", a quadrature bit is converted into 4 continuous sinusoidal waves in one symbol period. The relationship among $\varphi_{4}(n), \Delta \varphi_{4}(n)$ and $\operatorname{dat}_{4}[n]$ are as shown in Fig. 4(b).

Applying the OFDM-based PSDM-CL method in the DC microgrid and ignoring the sidebands, the spectrum of DC bus voltage $\nu_{\mathrm{PCC}}$ is shown in Fig. 5, where $f_{\mathrm{g}}$ is the grid frequency. The frequencies of the subcarriers are orthogonal to one another. They are all far apart from the power transmission band, the grid frequency $f_{\mathrm{g}}$ and the switching frequency $f_{\mathrm{s}}$, which alleviates the interference from power harmonics.

The data demodulation process of the PSDM-CL method includes two steps, as shown in Fig. 3. The first step is the 


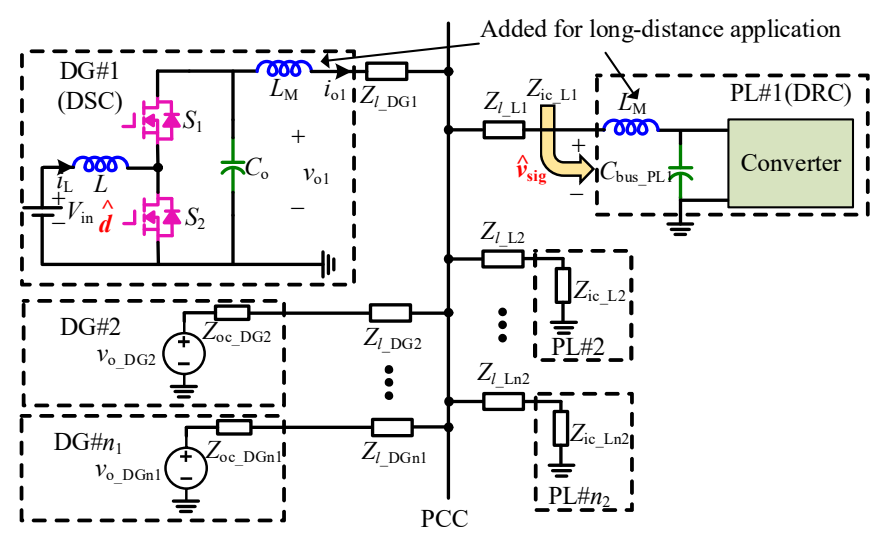

Fig. 6. Equivalent model of a typical DC microgrid.

Discrete Fourier Transform (DFT). In the DRC, the DC bus signal $v_{\mathrm{PCC}}$ is sent through a band pass filter (BPF) to eliminate interference. For the $k^{\text {th }}$ subcarrier, by performing DFT at $f_{\text {ck }}$ on the filtered signal $v_{\text {fil }}$, the phase in the $(n-1)^{\text {th }}$ and $n^{\text {th }}$ symbol period are calculated as $\theta_{\mathrm{k}}(n-1)$ and $\theta_{\mathrm{k}}(n)$ respectively, so the phase difference of the two symbols is

$$
\Delta \theta_{\mathrm{k}}(n)=\theta_{\mathrm{k}}(n)-\theta_{\mathrm{k}}(n-1) .
$$

Based on QDPSK principle, $\operatorname{dat}_{\mathrm{k}}[n]$ is got according to $\Delta \theta_{\mathrm{k}}(n)$. Finally, parallel-to-serial conversion is performed on all the received data of subcarriers, and the data can be decoded by the DRC.

\section{MODELING OF DC MICROGRID APPLYING THE PROPOSED METHOD}

In general, the characteristics of the communication channel would affect the performances of communication system. In the DC microgrid system, the impedance of the power electronic converter is the key determinant for the channel characteristics. Thus, it is necessary to model the DC microgrid system and analyze the impedance of the communication channel.

Considering the cable impedance, the equivalent model of a typical DC microgrid system is shown in Fig. 6. The system consists of $n_{1}$ DGs and $n_{2}$ PLs, and PCC is the point of common coupling in the system. $Z_{l_{-} \text {DGi }}$ and $Z_{l_{-} \mathrm{Lj}}$ are the equivalent cable impedance from DG $\# i$ and PL $\# j$ to PCC respectively. The DGs can be simplified by using Thevenin's equivalent theorem, as shown in Fig. 6 , where $v_{\mathrm{o}_{-} \mathrm{DGi}}$ and $Z_{\mathrm{oc} \text { DGi }}$ are the voltage source and output impedance of DG $\# i$ in Thevenin equivalent model, respectively. For PL $\# j$, the closed-loop input

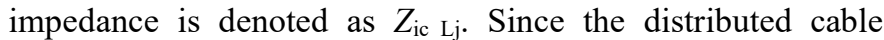
capacitance is small, it is merged to the input and output impedance of DGs and PLs.

For convenience, assume that the DC microgrid operates in island mode without grid-connected inverters. Suppose DG \#1, which is a droop-controlled boost converter, performs as the $\mathrm{DSC}$, and $\mathrm{PL} \# 1$, which is a buck converter, performs as the DRC. Compared with the power modulation signal, the communication carrier is a small perturbation. Since $f_{\mathrm{c}}$ is less than $f_{\mathrm{s}} / 5$, the traditional small signal analysis is applicable.

In the proposed method, the duty perturbation at $f_{\mathrm{c}}$ in power control loop corresponds to the amplitude of OFDM subcarriers with the same frequency. The signal transmission gain from

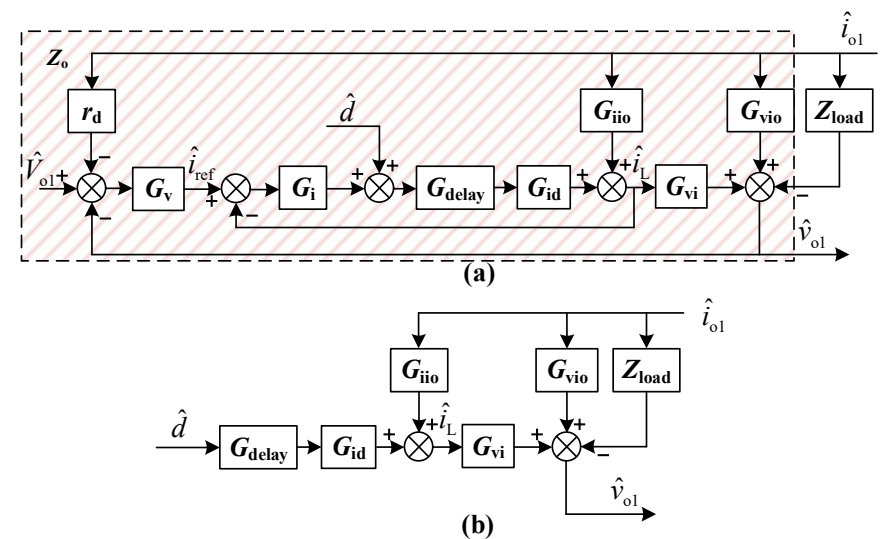

Fig. 7. Control block diagram of DG \#1 (a) Complete model. (b) Simplified model.

DSC to DRC, is defined as $G_{\text {sig }}$ in (7), where $\hat{d}$ is the perturbation signal added to the control loop in DSC, and $\hat{v}_{\text {sig }}$ is the signal received by DRC. $G_{\text {sig }}$ is the most important parameter for the PSDM-CL system and will be analyzed in detail.

$$
G_{\text {sig }}(s)=\frac{\hat{v}_{\text {sig }}}{\hat{d}}
$$

The detailed control block diagram of DG \#1 is illustrated in Fig. 7(a), where $\widehat{V}_{\text {o1 }}$ is the reference voltage without load, and $r_{\mathrm{d}}$ is the droop coefficient. The control scheme consists of three loops, the drooped-control outer loop, the voltage loop and the inductor current inner loop. The related transfer functions (TFs) are listed as follows.

$G_{\mathrm{v}}$ : compensation TF of voltage loop.

$G_{\mathrm{i}}$ : compensation TF of inductor current loop.

$G_{\text {delay }}:$ TF of the digital control delay link.

$G_{\text {idd }}$ TF from the duty cycle to inductor current $\hat{i}_{\mathrm{L}}$.

$G_{\text {iio }}$ : TF from $\hat{i}_{\mathrm{o} 1}$ to $\hat{i}_{\mathrm{L}}$.

$G_{\text {vio: }}$ TF from $\hat{i}_{\mathrm{o} 1}$ to $\hat{v}_{\mathrm{o} 1}$.

$G_{\mathrm{vi}}:$ TF from $\hat{i}_{\mathrm{L}}$ to $\hat{v}_{\mathrm{o} 1}$.

Referring to [24], the expressions of $G_{\mathrm{id}}, G_{\mathrm{iio}}, G_{\mathrm{vio}}$, and $G_{\mathrm{vi}}$ are calculated as in (8), where $D_{\mathrm{p}}$ is the duty cycle of $S_{2}$ in steady state, $I_{\mathrm{o} 1}$ and $V_{\mathrm{o} 1}$ are the DC output current and voltage of DG \#1.

$$
\left\{\begin{array}{c}
G_{\mathrm{id}}(s)=\frac{s C_{\mathrm{o}} V_{\mathrm{o} 1}+I_{\mathrm{o} 1}}{s^{2} L C_{\mathrm{o}}+\left(1-D_{\mathrm{p}}\right)^{2}} \\
G_{\mathrm{iio}}(s)=\frac{1-D_{\mathrm{p}}}{s^{2} L C_{\mathrm{o}}+\left(1-D_{\mathrm{p}}\right)^{2}} \\
G_{\mathrm{vi}}(s)=\frac{-s L_{\mathrm{L}}+V_{\mathrm{in}}}{s C_{\mathrm{o}} V_{\mathrm{o} 1}+I_{\mathrm{o} 1}} \\
G_{\mathrm{vio}}(s)=\frac{-V_{\mathrm{o} 1}}{s C_{\mathrm{o}} V_{\mathrm{o} 1}+I_{\mathrm{o} 1}}
\end{array}\right.
$$

The output impedance of DG \#1 can be expressed as (9), where $T_{\mathrm{v}}$ and $T_{\mathrm{i}}$ are the open-loop gain of the voltage and current control loop, respectively.

$$
\begin{gathered}
Z_{\mathrm{oc} \_ \text {DG1 }}=\frac{r_{\mathrm{d}_{\mathrm{v}} T_{\mathrm{v}}-G_{\mathrm{vi}_{\mathrm{o}}}\left(1+T_{\mathrm{i}}\right)-G_{\mathrm{iio}_{\mathrm{o}}} G_{\mathrm{vi}}}}{1+T_{\mathrm{i}}+T_{\mathrm{v}}} \\
T_{v}=G_{\nu} G_{i} G_{\text {delay }} G_{i d} G_{v i} \\
T_{i}=G_{i} G_{\text {delay }} G_{i d}
\end{gathered}
$$




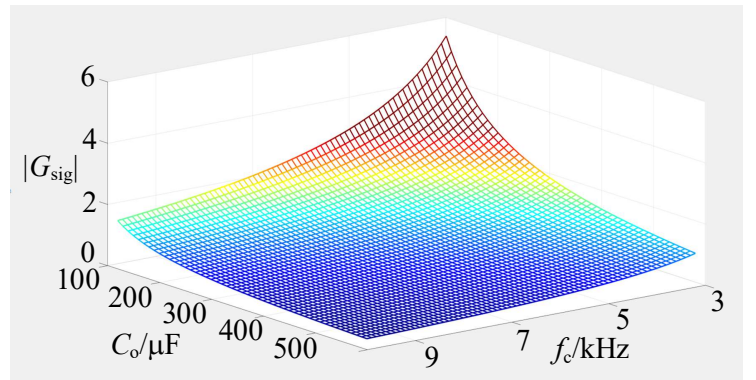

Fig. 8. Relationship among $\left|G_{\text {sig }}\right|, C_{o}$ and $f_{\mathrm{c}}$ ignoring line impedance.

For DG $\# i$ and PL $\# j$, the equivalent impedance $Z_{\mathrm{DGi}}$ and $Z_{\mathrm{Lj}}$ seen from PCC are

$$
\begin{gathered}
Z_{\text {DGi }}=Z_{l_{-} \text {DGi }}+Z_{\text {oc_DGi }}, \\
Z_{\mathrm{Lj}}=Z_{l_{-} \mathrm{Lj}}+Z_{\mathrm{ic} \_ \text {Lj }} .
\end{gathered}
$$

Therefore, the equivalent input impedance of the rest converters seen from PCC is

$$
Z_{\mathrm{PCC}}=1 /\left(\sum_{i=2}^{n_{1}} 1 / Z_{\mathrm{DGi}}+\sum_{j=1}^{n_{2}} 1 / Z_{\mathrm{Lj}}\right)
$$

So, the load impedance of DG \#1 can be written as

$$
Z_{\text {load }}=Z_{\text {l_DG1 }}+Z_{\text {PCC }} \text {. }
$$

Since the frequency of data carrier $f_{\mathrm{c}}$ is beyond the cut-off frequency of power control loop $f_{\mathrm{p}}$, the branches of the feedback, including droop-control loop, voltage loop and current loop, can be ignored at $f_{\mathrm{c}}$. Thus, the control diagram can be simplified as Fig. 7(b), and the transfer function between $\hat{d}$ and $\hat{v}_{01}$ can be derived as

$$
\frac{\hat{v}_{\mathrm{ol}}}{\hat{d}}=G_{\text {delay }} G_{\mathrm{id}} G_{\mathrm{vi}} \text {. }
$$

The transfer function from $\hat{v}_{01}$ to $\hat{v}_{\text {sig }}$ is

$$
\frac{\hat{v}_{\text {sig }}}{\hat{v}_{\text {o1 }}}=\frac{\hat{v}_{\text {sig }}}{\hat{v}_{\mathrm{PCC}}} \frac{\hat{v}_{\mathrm{PCC}}}{\hat{v}_{\mathrm{o} 1}}=\frac{Z_{\text {ic } \_ \text {L1 }}}{Z_{l_{l} \mathrm{~L} 1}+Z_{\mathrm{ic} \_\mathrm{L} 1}} \frac{Z_{\mathrm{PCC}}}{Z_{\text {load }}} .
$$

By combining (16) and (17), the signal transmission gain is

$$
G_{\text {sig }}(s)=\frac{\hat{v}_{\text {sig }}}{\hat{d}}=\frac{Z_{\text {ic } \_ \text {L1 }} Z_{\mathrm{PCC}} G_{\text {delay }} G_{\text {id }} G_{\mathrm{vi}}}{\left(Z_{l \_L}+Z_{\text {ic } \_ \text {L } 1}\right) Z_{\text {load }}} .
$$

Ignoring the line impedance, (18) can be simplified as

$$
G_{\text {sig }}(s)=G_{\text {delay }} \frac{-s L I_{\mathrm{L}}+V_{\text {in }}}{s^{2} L C_{0}+\left(1-D_{\mathrm{p}}\right)^{2}} .
$$

It can be deduced from (19) that $\left|G_{\text {sig }}\right|$ is determined by the inductance $L$ and capacitance $C_{\mathrm{o}}$ of DG \#1 at a fixed output power. In the proposed system, capacitor $C_{\mathrm{o}}$ should be designed to fit for the required signal strength. The relationship among $\left|G_{\text {sig }}\right|$, capacitance $C_{\mathrm{o}}$ and carrier frequency $f_{\mathrm{c}}$ are shown in Fig. 8. It can be observed that $\left|G_{\text {sig }}\right|$ decreases as $f_{\mathrm{c}}$ increases, which corresponds with the characteristics of output LPF. Also, at a given $f_{\mathrm{c}},\left|G_{\mathrm{sig}}\right|$ is negatively correlated with $C_{\mathrm{o}}$, which indicates that reducing the capacitance of the converter at the bus port could improve the signal transmission gain.

However, in most applications of DC microgrids, the line impedance cannot be ignored. In this paper, the impedance of the power line is assumed to be

$$
Z_{l_{-} \mathrm{PL} 1}=\left(0.009+3 * 10^{-7} s\right)^{*} l_{\mathrm{PL} 1},
$$

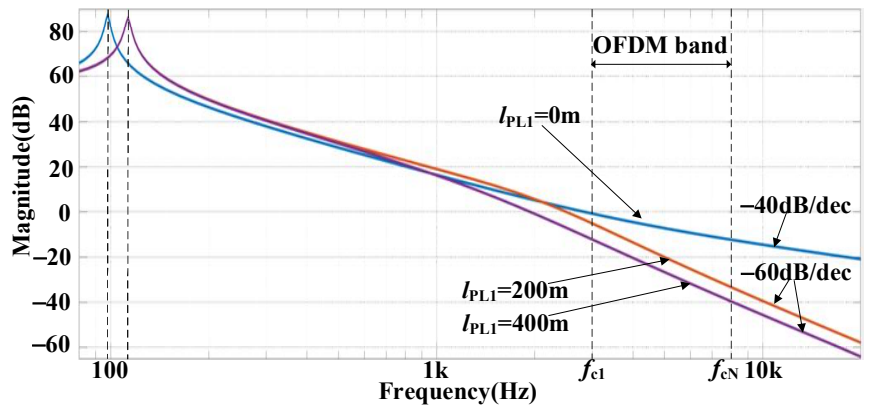

Fig. 9. Gain-frequency diagram of $G_{\text {sig }}(s)$ with different power line length.

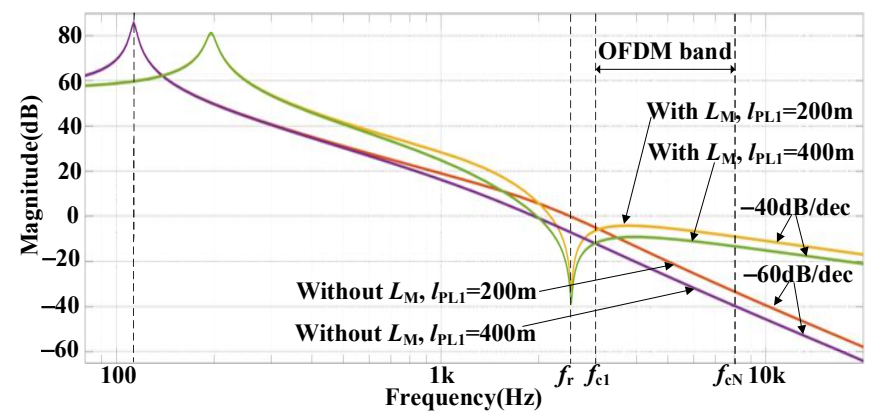

Fig. 10. Gain-frequency diagram of $G_{\text {sig }}(s)$ with and without $L_{\mathrm{M}}$.

where $l_{\mathrm{PL} 1}$ is the length of the power line in meters, the gain-frequency characteristics of $G_{\text {sig }}$ with different $l_{\text {PL1 }}$ is plotted in Fig. 9. It can be observed that the communication signal is reduced with the increasing of $l_{\mathrm{PL} 1}$. In addition, the decay rate of $\left|G_{\text {sig }}\right|$ is about $60 \mathrm{~dB} / 10 \mathrm{dec}$ in frequency domain. Thus, the higher-frequency subcarriers would be attenuated more seriously in the communication channel.

In order to increase the signal transmission gain of high-frequency subcarriers in long-distance applications, an inductor $L_{\mathrm{M}}$ can be added at the bus port of the converter, as suggested in Fig. 6. The existence of $L_{\mathrm{M}}$ increases $Z_{\mathrm{ic} \_\mathrm{L} 1}$ and thereby increasing $\left|G_{\text {sig }}\right|$ according to (18). Fig. 10 shows the gain-frequency characteristics with added $L_{\mathrm{M}}$. It can be observed that $L_{\mathrm{M}}$ promotes the signal transmission gain at high frequencies. However, an additional zero at $f_{\mathrm{r}}$ is introduced by the resonance of $L_{\mathrm{M}}$ and the input capacitor $C_{\text {bus_pL1 }}$ of DRC. To reduce the impact on the communication, $f_{\mathrm{r}}$ should be assigned to a frequency lower than the OFDM spectrum. Besides, the power transmission band is typically from DC to several hundred hertz. In order to reduce the impact on power control, it is preferable to set $f_{\mathrm{r}}$ higher than the power transmission band. The detailed method is out of the scope of this paper, and will not be discussed.

\section{COMMUNICATION SYSTEM CONSIDERATIONS}

\section{A. Frame Format Design}

According to the above analysis, the transmission gain and delays of subcarriers in the channel are different, which causes ISI and inter-carrier interference (ICI). In OFDM technology, a cyclic prefix (CP) is usually added to eliminate ICI, reduce ISI and improve the signal-to-noise ratio (SNR). This method is adopted by this paper, and the communication frame format is shown in Fig. 11. For simplicity, only one subcarrier is plotted. Each frame format consists of one synchronization symbol and $N_{\text {data }}$ data symbols. The synchronization symbol and the data 


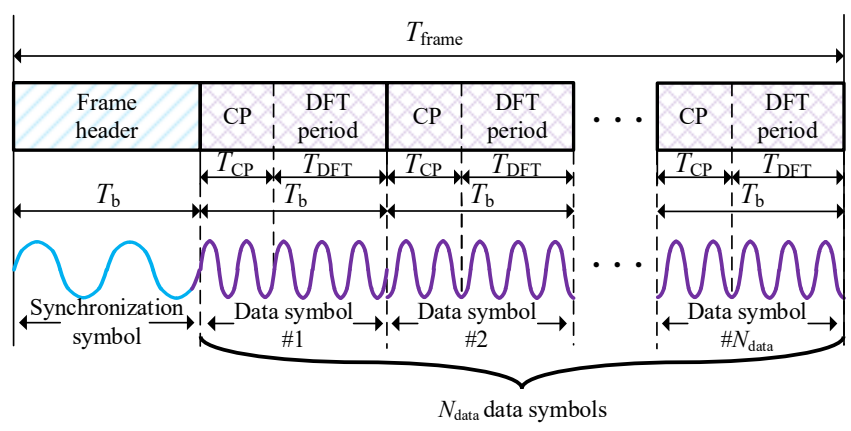

Fig. 11. Structure of frame format.

symbol (including CP) have the same duration, which is $T_{\mathrm{b}}$. The synchronization symbol performs as the frame header, marking the beginning of a frame. The data is firstly distributed to $N$ subcarriers, and then modulated into the periods of data symbols according to QDPSK principle, as discussed in Section II. The detailed communication process is illustrated as follows.

First, the synchronization symbol is sent from the transmitter to the receiver. The carrier frequency used by the synchronization bit is generally selected among OFDM subcarrier frequencies $f_{\mathrm{c} 1} \sim f_{\mathrm{cN}}$. The receiver detects the amplitude of the synchronization signal through a sliding window DFT algorithm to achieve frame synchronization.

Then, $N_{\text {data }}$ data symbols are transmitted in sequence. Each data symbol is composed of a CP and a DFT period, denoted as $T_{\mathrm{CP}}$ and $T_{\mathrm{DFT}}$ respectively. The lengths of CP and DFT period should satisfy

$$
T_{\mathrm{b}}=T_{\mathrm{CP}}+T_{\mathrm{DFT}} .
$$

The phase of each subcarrier is continuous during $T_{\mathrm{CP}}$ and $T_{\mathrm{DFT}}$, which ensures the correctness of demodulation results even if the DFT window at the receiver is not completely aligned with $T_{\mathrm{DFT}}$.

The performance of OFDM communication is greatly affected by system parameters, including subcarrier frequency spacing $\Delta f_{\mathrm{c}}$ and cyclic prefix length $T_{\mathrm{CP}}$.

In general, the subcarrier spacing $\Delta f_{\mathrm{c}}$ is set with respect to $T_{\mathrm{DFT}}$, which is

$$
\Delta f_{\mathrm{c}}=\frac{M_{\mathrm{s}}}{T_{\mathrm{DFT}}},
$$

where $M_{\mathrm{s}}$ is a positive integer. In order to achieve maximum band utilization in a narrow band, $M_{\mathrm{s}}$ is set to 1 in the proposed method, thus $\Delta f_{\mathrm{c}}$ is $1 / T_{\mathrm{DFT}}$.

In order to eliminate ICI caused by the multipath delay of subcarriers in the communication channel, $T_{\mathrm{CP}}$ must be greater than the maximum multipath delay, which is

$$
T_{\mathrm{CP}}>\max \left\{\frac{1}{2 \pi f_{\mathrm{c}}} \angle G_{\mathrm{sig}}\left(2 \pi f_{\mathrm{c}}\right)\right\}-\min \left\{\frac{1}{2 \pi f_{\mathrm{c}}} \angle G_{\text {sig }}\left(2 \pi f_{\mathrm{c}}\right)\right\} \text {. }
$$

\section{B. ISI Evaluation}

Conventionally, ISI for a communication system is analyzed and evaluated in time domain, which is rather complicated. In order to deal with different and complex communication channel characteristics in the power electronics systems with the proposed OFDM-based PSDM-CL technique, a practical ISI evaluation method is designed as following.

In an OFDM system, the subcarrier $x_{\mathrm{k}}$ in the $n^{\text {th }}$ symbol period suffers interference from previous symbols due to the

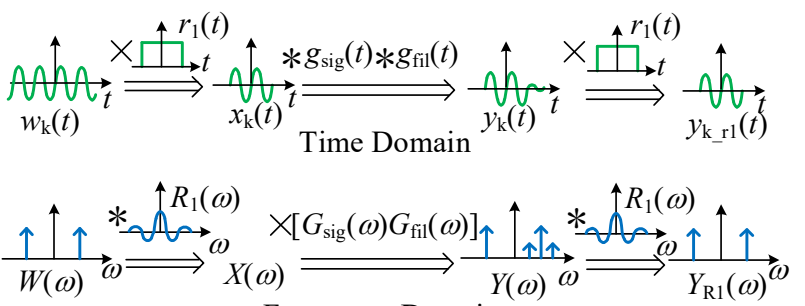

Frequency Domain

Fig. 12. Signal transformation process in time and frequency domain.

non-ideal characteristic of the communication channel, which is defined as ISI. For subcarrier $x_{\mathrm{k}}$, ISI originates from two parts, which are interference from itself and from other subcarriers. The latter can be easily eliminated by adding a $\mathrm{CP}$, and this paper will focus on evaluating ISI from itself.

In general, the ISI is mainly from adjacent symbol. In the $n^{\text {th }}$ symbol period, the $k^{\text {th }}$ subcarrier signal $x_{\mathrm{k}}(t)$ to be transmitted is the result of multiplying the ideal sine wave $w_{\mathrm{k}}(t)$ and the rectangular window $r_{1}(t)$ in time domain, as shown in Fig. 12.

$$
x_{\mathrm{k}}(t)=w_{\mathrm{k}}(t) r_{1}(t)
$$

The expression of $r_{1}(t)$ is

$$
r_{1}(t)=\left\{\begin{array}{c}
1,-\frac{T_{\mathrm{DFT}}}{2}<t<\frac{T_{\mathrm{DFT}}}{2} \\
0, t \geq-\frac{T_{\mathrm{DFT}}}{2} \text { or }, t<\frac{T_{\mathrm{DFT}}}{2}
\end{array} .\right.
$$

The TF of the channel is composed of signal transmission gain $G_{\text {sig }}(\omega)$ and the signal filter $G_{\text {fil }}(\omega)$ at the receiver. Passing through the channel, the received signal $y_{\mathrm{k}}(t)$ is

$$
y_{\mathrm{k}}(t)=F^{-1}[Y(\omega)]=F^{-1}\left\{F\left[x_{\mathrm{k}}(t)\right] G_{\mathrm{sig}}(\omega) G_{\mathrm{fil}}(\omega)\right\} .
$$

By performing DFT algorithm on $y_{\mathrm{k}}(t)$, the demodulated results at $f_{\text {ck }}$ in the $n^{\text {th }}$ symbol period can be calculated as

$$
Y_{\mathrm{R} 1}(\omega)=Y(\omega) * R_{1}(\omega)
$$

The $n^{\text {th }}$ symbol will interfere the demodulation of the $(n+1)^{\text {th }}$ symbol. By adding a delayed rectangular window $r_{2}(t)$ to $y_{\mathrm{k}}(t)$ and performing DFT, the received signal can be calculated as

$$
Y_{\mathrm{R} 2}(\omega)=F\left[y_{\mathrm{k}_{-} 2}(t)\right]=F\left[y_{\mathrm{k}}(t) r_{2}(t)\right]=Y(\omega) * R_{2}(\omega) .
$$

The relationship between $r_{2}(t)$ and $r_{1}(t)$ is

$$
r_{2}(t)=r_{1}\left(t-T_{\mathrm{b}}\right) \text {. }
$$

The analytical expressions of (27) and (28) are often complex or even unsolvable. Therefore, in practical, numerical calculation tools such as MATLAB can be used to calculate $Y_{\mathrm{R} 1}(\omega)$ and $Y_{\mathrm{R} 2}(\omega)$. Define $\alpha$ as

$$
\alpha=\frac{\left|Y_{\mathrm{R} 2}(\omega)\right|}{\left|Y_{\mathrm{R} 1}(\omega)\right|} .
$$

Obviously $\alpha$ represents the severity of ISI. The higher the value of $\alpha$, the more serious the ISI problem will be. The ISI of the proposed system will be evaluated in Section V.A by calculating $\alpha$ and the maximum angle error $\theta_{\mathrm{e}}$.

\section{EXPERIMENTAL RESULTS}

\section{A. Experimental Setup}

The feasibility of the PSDM-CL method and the correctness of the theoretical analysis have been experimentally verified on a $2 \mathrm{~kW} \mathrm{DC} \mathrm{microgrid} \mathrm{experimental} \mathrm{platform.} \mathrm{The} \mathrm{platform}$ 


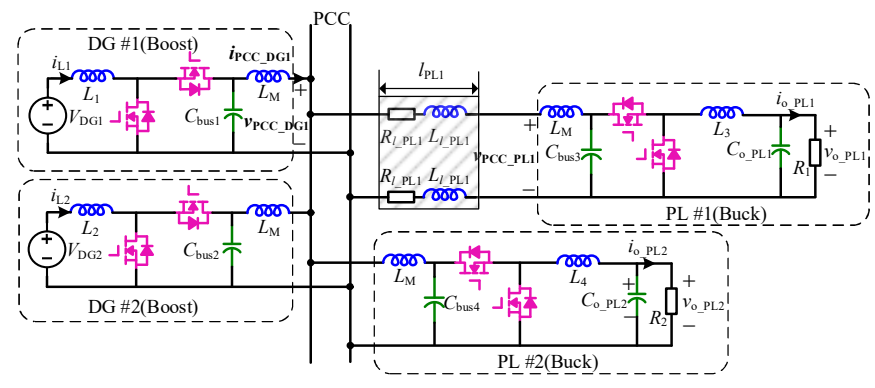

Fig. 13. Structure of experimental platform.

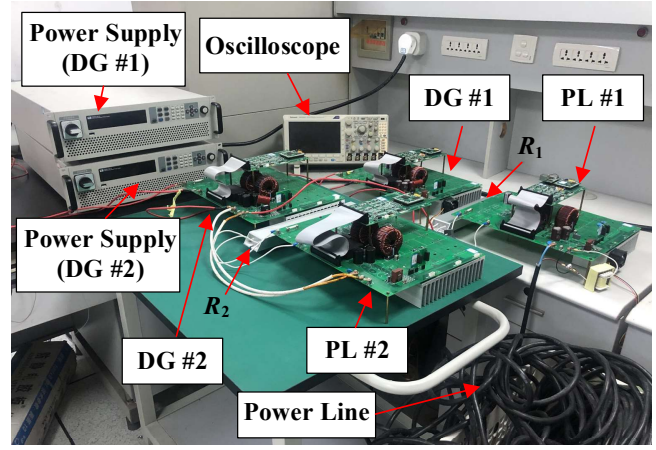

Fig. 14. Photo of experimental platform.

TABLE.I

PARAMETERS OF DC MICROGRID SYSTEM

\begin{tabular}{|c|c|c|}
\hline Description & Symbol & Value \\
\hline Input voltage of DG \#1, DG \#2 & $V_{\mathrm{DG} 1}, V_{\mathrm{DG} 2}$ & $120-200 \mathrm{~V}$ \\
\hline Bus voltage reference without load & $\widehat{V}_{\mathrm{o} 1}$ & $380 \mathrm{~V}$ \\
\hline Output voltage of PL \#1, PL \#2 & $V_{\mathrm{o} \text { PL1 }}, V_{\mathrm{o} \text { PL2 }}$ & $150-200 \mathrm{~V}$ \\
\hline Load of PL \#1, PL \#2 & $R_{1}, R_{2}$ & $40 \Omega$ \\
\hline Droop coefficient & $r_{\mathrm{d}}$ & $3 \mathrm{~V} / \mathrm{A}$ \\
\hline Switching frequency & $f_{\mathrm{s}}$ & $50 \mathrm{kHz}$ \\
\hline Output capacitance of DG \#1, DG \#2 & $C_{\text {bus } 1}, C_{\text {bus } 2}$ & $100 \mu \mathrm{F}$ \\
\hline Input capacitance of PL \#1, PL \#2 & $C_{\text {bus } 3}, C_{\text {bus } 4}$ & $100 \mu \mathrm{F}$ \\
\hline Output capacitance of PL \#1, PL \#2 & $C_{\mathrm{o}_{\mathrm{P}} \mathrm{PL} 1}, C_{\mathrm{O} \_ \text {PL2 }}$ & $300 \mu \mathrm{F}$ \\
\hline Inductance & $L_{1}, L_{2}, L_{3}, L_{4}$ & $2 \mathrm{mH}$ \\
\hline Power line length between PCC and PL \#1 & $l_{\mathrm{PL} 1}$ & $1-200 \mathrm{~m}$ \\
\hline Inductance for impedance matching & $L_{\mathrm{M}}$ & $40 \mu \mathrm{H}$ \\
\hline \multicolumn{3}{|l|}{ TABLE II } \\
\hline Description & Parameters & Value \\
\hline Number of subcarriers & $N$ & 6 \\
\hline Frequencies of subcarriers & $f_{\mathrm{cl}}-f_{\mathrm{cN}}$ & $3-8 \mathrm{kHz}$ \\
\hline Symbol period & $T_{\mathrm{b}}$ & $1.2 \mathrm{~ms}$ \\
\hline CP period & $T_{\mathrm{CP}}$ & $0.2 \mathrm{~ms}$ \\
\hline DFT period & $T_{\mathrm{DFT}}$ & $1 \mathrm{~ms}$ \\
\hline Communication baud rate & $R_{\mathrm{B}}$ & $9.6 \mathrm{kbps}$ \\
\hline Frequency of the synchronization symbol & $f_{\text {sych }}$ & $3 \mathrm{kHz}$ \\
\hline Number of data symbols in one frame & $N_{\text {data }}$ & 9 \\
\hline Frame duration & $T_{\text {frame }}$ & $12 \mathrm{~ms}$ \\
\hline
\end{tabular}

structure and photo are shown in Fig. 13 and Fig. 14, respectively. The DC microgrid includes two DGs with boost topology and two PLs with buck topology. DG \#1 performs as DSC and PL \#1 performs as DRC. TMS320F28377 from Texas Instruments is used for each power converter to implement the control algorithm and the proposed OFDM-based PSDM-CL method. 200-meters cables are used to connect PCC and PL \#1 to test the communication performance with long cables.

The experimental parameters of the DC microgrid and the communication system are provided in Table I and Table II, respectively. It should be noted that $L_{\mathrm{M}}$ is only for the experiment in Section V. D. In this platform, the converter's power control loop cut-off frequency is set to $2 \mathrm{kHz}$, while the
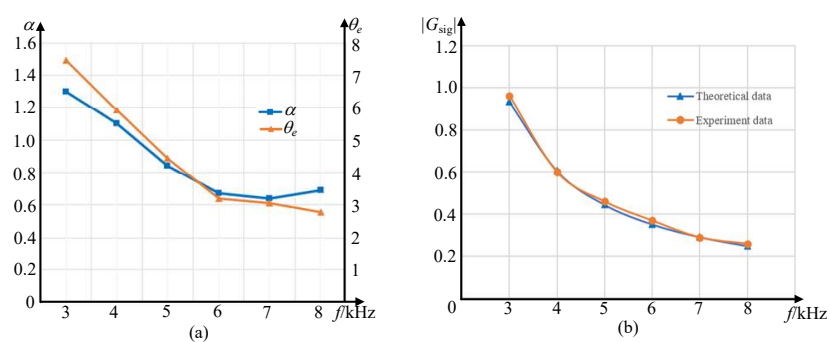

Fig. 15. (a) Simulation results of ISI evaluation. (b) Comparison of theoretical and experimental $\left|G_{\text {sig }}\right|$.

communication carrier frequencies are selected above $3 \mathrm{kHz}$ to decouple power conversion and data communication.

In order to meet the communication rate requirement of secondary control in DC microgrid, the DFT period is set to $1 \mathrm{~ms}$. Besides, the frame duration is $12 \mathrm{~ms}$, which is much less than $100 \mathrm{~ms}$, i.e., the real-time transmission requirement of secondary control. According to (23), the maximum multipath delay is $0.089 \mathrm{~ms}$. Thus, a cyclic prefix $T_{\mathrm{CP}}$ is set to $0.2 \mathrm{~ms}$ to eliminate ICI. Six subcarriers $x_{1} \sim x_{6}$ are used for OFDM modulation on DG \#1 and each subcarrier is modulated by QDPSK. Thus, each subcarrier in one frame contains 16-bits data information according to QDPSK principle. The communication baud rate of the experimental platform is

$$
R_{\mathrm{B}}=\frac{6 \times \log _{2} 4}{1.2 \times 10^{-3}}=9.6 \mathrm{kbps} \text {. }
$$

Before the experimental study, a MATLAB/Simulink model of the communication system is built to evaluate ISI based on Section IV. C. Based on the simulation results, the relationship among $\alpha, \theta_{\mathrm{e}}$ and $f_{\mathrm{ck}}$ is plotted in Fig. 15(a). It can be observed that the severity of ISI decreases as $f_{\mathrm{c}}$ increases. The maximum angle error caused by ISI is $7.56^{\circ}$, which is much smaller than $45^{\circ}$. Hence, the SNR can be guaranteed when QDPSK is applied in the experiment.

\section{B. System Modeling Experimental Verification}

This experiment aims to check the correctness of system modeling in Section III. In this test, the length of the cable $l_{\mathrm{PL} 1}$ is 1 meter. The peak value of $\hat{v}_{\text {sig }}$ is measured at PL \#1 when perturbation $\hat{d}$ is added to the control loop in DG \#1. The theoretical and experimental results of $\left|G_{\text {sig }}\right|$ are calculated and plotted, as shown in Fig. 15(b). It can be observed that the experimental result is almost in line with the theoretical one, which verifies the correctness of the system modeling. It is also clear that $\left|G_{\text {sig }}\right|$ gradually decreases as the frequency increases. In order to compensate for the attenuation of higher-frequency subcarriers, a viable option is to increase the perturbation amplitudes of higher-frequency subcarriers.

\section{System Performance Test with Short Cable}

In this experiment, the power transfer and communication functions of the DC microgrid system are tested. The length of the cable $l_{\mathrm{PL} 1}=1 \mathrm{~m}$. The DC microgrid is operated in steady state. DG \#1 and DG \#2 are transferring a total power of $2 \mathrm{~kW}$ to the PLs, and each PL consumes a fixed power of $1 \mathrm{~kW}$.

In this case, DG \#1 sends a set of test data dat $_{\mathrm{k}}[8]_{1}$ repeatedly to other power converters via the DC bus. The subcarrier frequency $f_{\mathrm{ck}}$, perturbance amplitude $d_{\mathrm{k}}$ and quaternary data sent by each subcarrier are shown in Table III. According to (16), the maximum perturbation of $v_{\mathrm{PCC}}$ introduced by the 
TABLE III

PARAMETERS OF SUBCARRIERS IN SECTION V.C

\begin{tabular}{cccc}
\hline Subcarrier & $\boldsymbol{f}_{\text {ck }}$ & $\boldsymbol{d}_{\mathbf{k}}$ & $\boldsymbol{d a t}_{\mathbf{k}}[\mathbf{8}]_{\mathbf{1}}$ \\
\hline$x_{1}$ & $3 \mathrm{kHz}$ & 0.028 & 01112110 \\
$x_{2}$ & $4 \mathrm{kHz}$ & 0.032 & 22102012 \\
$x_{3}$ & $5 \mathrm{kHz}$ & 0.032 & 11112222 \\
$x_{4}$ & $6 \mathrm{kHz}$ & 0.034 & 01332330 \\
$x_{5}$ & $7 \mathrm{kHz}$ & 0.036 & 23121032 \\
$x_{6}$ & $8 \mathrm{kHz}$ & 0.038 & 01233210 \\
\hline
\end{tabular}

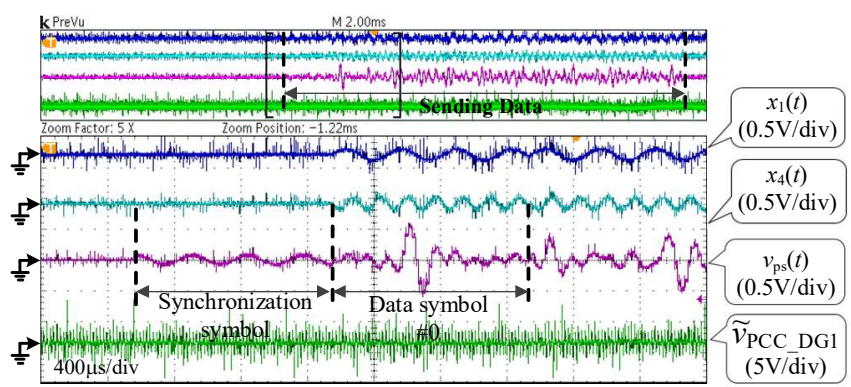

Fig. 16. Modulation waveforms of DG \#1.

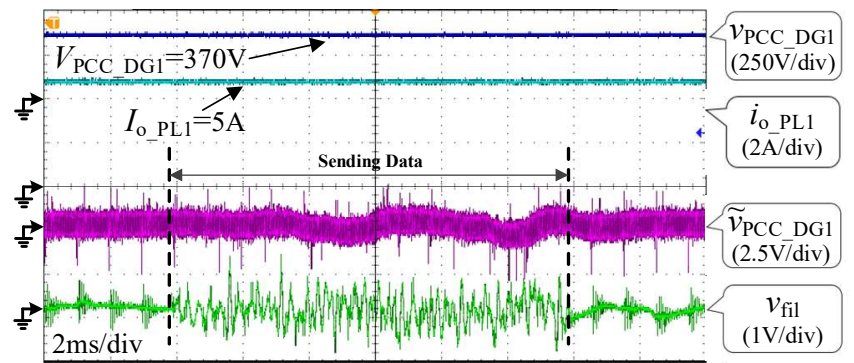

Fig. 17. Waveforms of DC microgrid system applying PSDM-CL method.

communication is $489 \mathrm{mV}$. This value meets the power quality requirements of the DC microgrid.

Fig. 16 depicts the modulation waveforms of DG \#1 when it is sending $d a t_{\mathrm{k}}[8]_{1} . x_{1}(t)$ and $x_{4}(t)$, which are the first and fourth subcarrier modulation waveforms respectively, are expressed by the outputs of digital-to-analog conversion (DAC) module. $v_{\mathrm{ps}}(t)$ is the DAC output of $s(t)$, which has been explained in Section II. $\tilde{v}_{\text {PCC_DG1 }}$ is the ac component of $\nu_{\text {PCC_DG1 }}$. It can be observed that the carrier frequency used by the synchronization symbol is $3 \mathrm{kHz}$, and the waveforms of $x_{1}(t), x_{4}(t)$ and $v_{\mathrm{ps}}(t)$ are matched with the theory in Section II.

Fig. 17 demonstrates the operation states of the DC microgrid. It can be observed that $V_{\text {PCC_DG1 }}$ is around $370 \mathrm{~V}$. The ac component $\tilde{v}_{\text {PCC DG1 }}$ has a peak-to-peak amplitude of around $1.2 \mathrm{~V}$. The load current of PL \#1 $I_{\mathrm{O}-\mathrm{PL} 1}$ is about $5 \mathrm{~A}$, which corresponds to $1 \mathrm{~kW}$ load. The fluctuation of $v_{\text {PCC_DG1 }}$ introduced by communication does not exceed $1 \mathrm{~V}$. The filtered signal $v_{\text {fil }}$ is obtained by passing through a BPF from $v_{\text {PCC }}$ PLl, and it is ready for demodulation.

At PL \#1, demodulation algorithm is performed by the microcontroller, and the results of the subcarrier data are expressed by the outputs of DAC module, as shown in Fig. 18. The results are consistent with Table III, which proves the effectiveness of the proposed communication method. The signals of $\mathrm{CH} 2 \sim \mathrm{CH} 7$ lag about $T_{\mathrm{DFT}}$ behind the signal of $\mathrm{CH} 1$, which is because the controller needs to perform DFT within a complete $T_{\mathrm{DFT}}$. Besides, the frequency spectrum of $v_{\text {fil }}$ is shown in Fig. 19. The frequency band of the FFT results is distributed

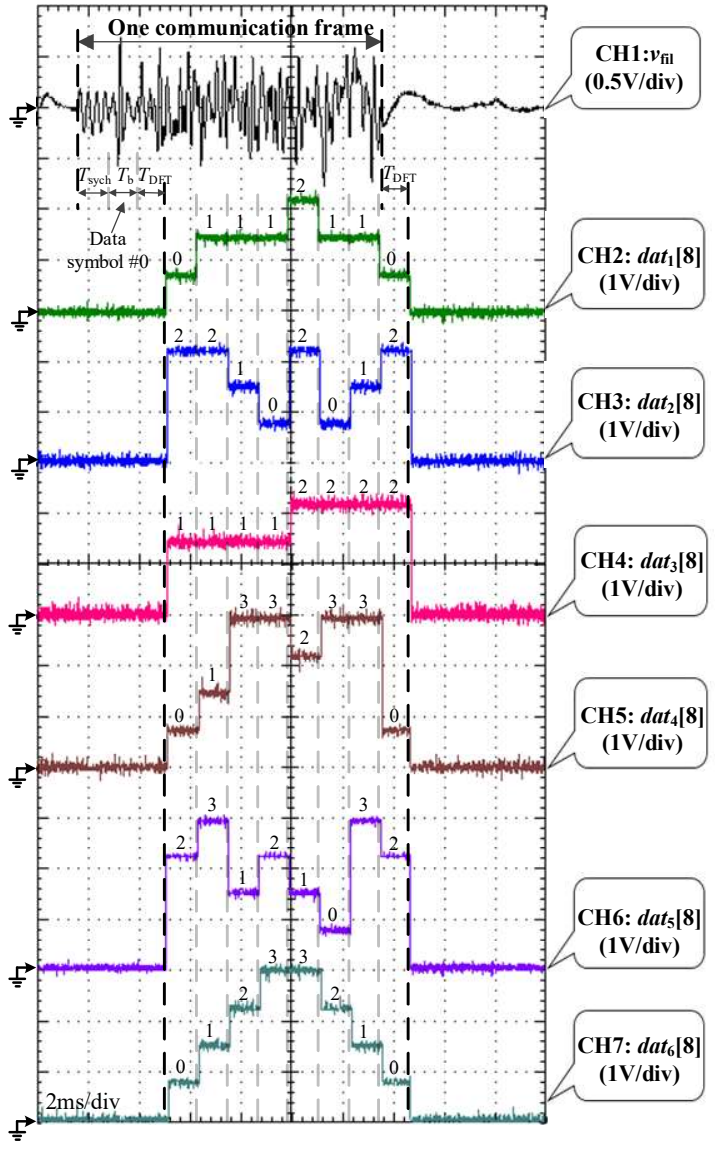

Fig. 18. Demodulation results at $\mathrm{PL} \# 1$ under $I_{\mathrm{PL} 1}=1 \mathrm{~m}$.

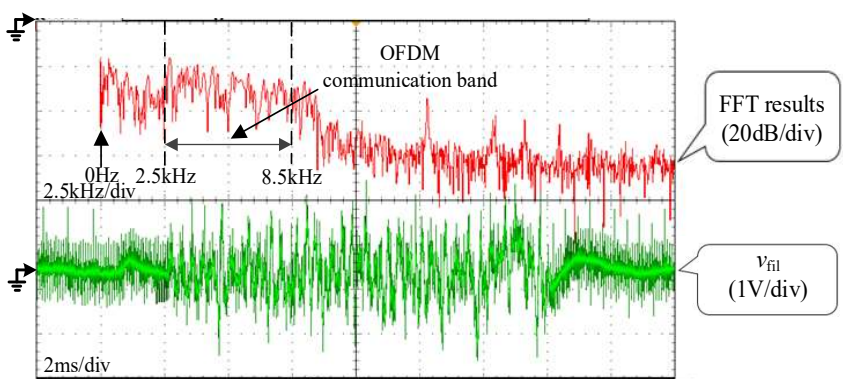

Fig. 19. Spectrum analysis results of filter output signal.

between $2.5 \mathrm{kHz}$ and $8.5 \mathrm{kHz}$. The frequency band is relatively flat, which verifies the relevant analysis in Section III.

D. System Performance Test with Long Cable

This experiment aims to test the communication function under the circumstances of long transmission distance. In this case, $l_{\mathrm{PL} 1}$ is set to $200 \mathrm{~m}$. In order to increase the input impedance of converters, $L_{\mathrm{M}}$ is added at the bus port of every converter.

As an example, a simple communication protocol is designed. In the protocol, subcarrier $x_{1}$ and $x_{2}$ are arranged to represent the command type and value respectively, while subcarrier $x_{3}$ is reserved for the summation check results of $x_{1}$ and $x_{2}$. Subcarrier $x_{4}, x_{5}$ and $x_{6}$ are not used in the simple protocol. They can be used in more complicated protocols for advanced DC microgrid secondary control.

In this experiment, DG \#1 performs as the master and sends a command frame to change the output power of PL \#1 by the 
TABLE IV

PARAMETERS OF SUBCARRIERS IN SECTION V.D

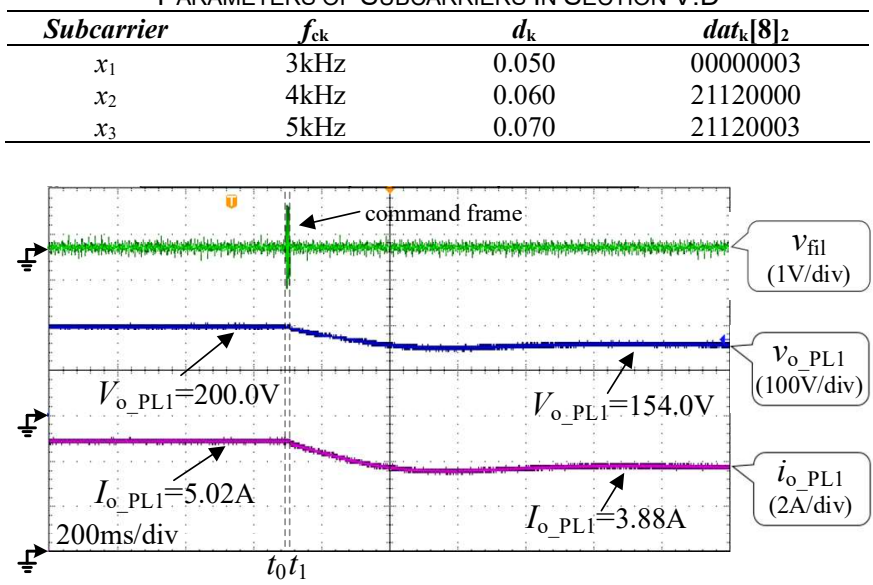

Fig. 20. Waveforms of PL \#1 voltage and current before and after communication.

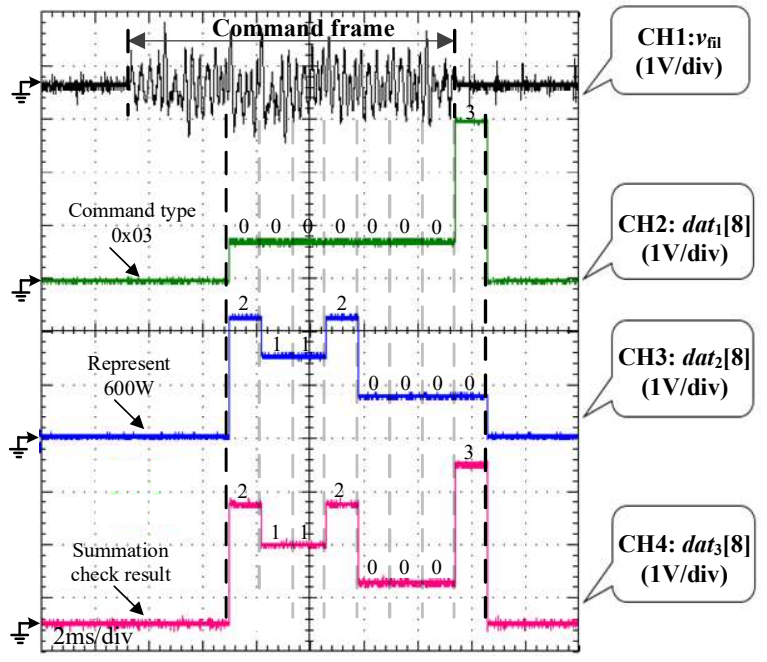

Fig. 21. Demodulation results at PL \#1 under $I_{P L 1}=200 \mathrm{~m}$.

proposed OFDM-based PSDM-CL method. The detailed parameters of the command frame are illustrated in Table IV. In order to promote the signal strength at DRC, $d_{\mathrm{k}}$ is set larger than that in the experiment of Section V.C. The command type $0 \mathrm{x} 03$ in the subcarrier $x_{1}$ represents that DG \#1 orders PL \#1 to regulate its output power to the given value. The given value is $600 \mathrm{~W}$, which is expressed by the subcarrier $x_{2}$. As the slave, PL \#1 samples the bus voltage, demodulates the data frame and regulates its output power according to the command.

Fig. 20 depicts the waveforms of the above process. $v_{\text {fil }}$ is the filtered signal obtained by passing through a BPF from $v_{\text {PCC_PL1. }}$ The initial operation state of DC microgrid is the same as that in Section V.C. The output power of PL \#1 is $1 \mathrm{~kW}$. At $t_{0}$, a command frame is sent by DG \#1. Starting from $t_{1}, \mathrm{PL} \# 1$ successfully receives the command frame and regulates its output power to $600 \mathrm{~W}$ according to the data information.

In order to clarify the details of the communication process, the command frame in Fig. 20 is zoomed in, along with its demodulation results, as shown in Fig. 21. The demodulation results are the same with $\operatorname{dat}_{\mathrm{k}}[8]_{2}$ in Table IV, which proves the feasibility of the proposed method under the circumstances of long transmission distance.

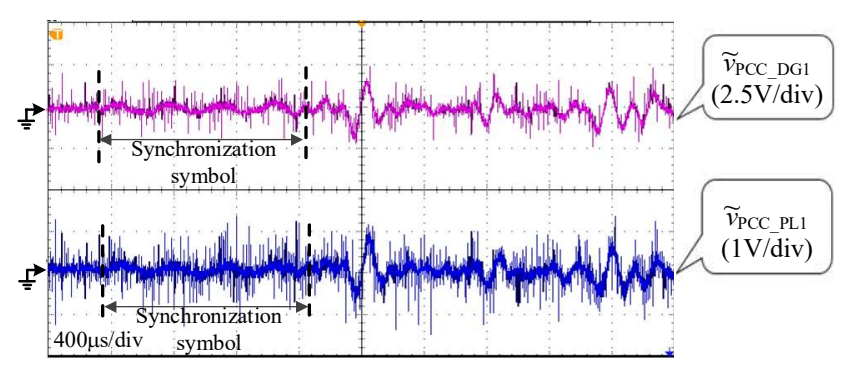

Fig. 22. Influence of power line impedance on communication.

Furtherly, the enlarged waveforms of $\tilde{v}_{\text {PCC_DG1 }}$ and $\tilde{v}_{\text {PCC_PL1 }}$ during the communication process are illustrated in Fig. 22. The ac amplitude of $v_{\text {pcc_PL1 }}$ is about $2 / 5$ that of $v_{\text {pcc_DG1, which }}$ is consistent with the analysis in Section III. Whereas, due to the existence of $L_{\mathrm{M}}$, the signal is still large enough to be correctly demodulated by the DRC.

\section{CONCLUSIONS \& FUTURE WORKS}

This paper analyzes the principles and implementations of OFDM-based PSDM-CL method and validates its feasibility in the DC microgrid. A practical evaluation method for ISI is designed, which can be promoted to all power electrical systems applying the OFDM-based PSDM-CL method. A communication rate of $9.6 \mathrm{kbps}$ has been achieved in a $2 \mathrm{~kW}$ DC microgrid, which meets the requirements of microgrid applications. Since no additional communication controllers and less communication components are required by the proposed method, it has the advantages of low costs and simple implementation, and therefore has broad application prospects.

Nevertheless, the proposed method introduced in this paper can be improved in many aspects. First, advanced techniques used in conventional OFDM, such as equalization and Peak-to-Average-Power-Ratio (PAPR) suppression techniques, can be adopted to deal with complicated operating conditions. Second, in practical, communication failure might occur due to complex power transfer situations. In this case, Automatic Repeat Request (ARQ) mechanism can be employed to deal with the communication failure. Third, in long-distance communication applications, additional impedance-match components are required to promote the signal transmission gain of high-frequency subcarriers, which is worth further research.

\section{REFERENCES}

[1] S. Al-Sarawi, M. Anbar, K. Alieyan, and M. Alzubaidi, "Internet of Things (IoT) communication protocols: review," in 2017 8th Int. Conf. on Information Technology (ICIT), pp. 685-690, May 2017.

[2] R. Ma, H. Chen, Y. Huang and W. Meng, "Smart grid communication: its challenges and opportunities," IEEE Trans. Smart Grid, vol. 4, no. 1, pp. 36-46, Mar. 2013.

[3] T. Dragičević et al., "DC microgrids-Part I: A review of control strategies and stabilization techniques," IEEE Trans. Power Electron., vol. 31, no. 7, pp. 4876-4891, July 2016.

[4] J. M. Guerrero et al., "Hierarchical control of droop-controlled AC and DC microgrids - A general approach toward standardization," IEEE Trans. Ind. Electron., vol. 58, pp. 158-172, Jan. 2011.

[5] V. Nasirian, S. Moayedi, A. Davoudi and F. L. Lewis, "Distributed cooperative control of DC microgrids," IEEE Trans. Power Electron., vol. 30, no. 4, pp. 2288-2303, April 2015. 
[6] X. Chen et al., "Distributed cooperative control of multiple hybrid energy storage systems in a DC microgrid using consensus protocol," IEEE Trans. Ind. Electron., vol. 67, no. 3, pp. 1968-1979, March 2020.

[7] X. Chen et al., "Distributed cooperative control and stability analysis of multiple DC electric springs in a DC microgrid," IEEE Trans. Ind. Electron., vol. 65, no. 7, pp. 5611-5622, July 2018.

[8] S. Sahoo, et al., "A cooperative adaptive droop based energy management and optimal voltage regulation scheme for DC Microgrids," IEEE Trans. Ind. Electron., vol. 67, no. 4, pp. 2894-2904, April 2020.

[9] M. Shi et al, "Distributed optimal control of energy storages in a DC microgrid with communication delay," IEEE Trans. Smart Grid, vol. 11, no. 3, pp. 2033-2042, May 2020.

[10] Y. Zhu et al. "Communication platform for energy management system in a master-slave control structure microgrid," in Proc. of the 7th Int. Power Electron. and Motion Control Conf., pp. 141-145, 2012.

[11] S. Moon, S. Yoon and J. Park, "A new low-cost centralized MPPT controller system for multiply distributed Photovoltaic power conditioning modules," IEEE Trans. Smart Grid, vol. 6, no. 6, pp. 2649-2658, Nov. 2015.

[12] H. Liang et al., "Stability enhancement of decentralized inverter control through wireless communications in microgrids," IEEE Trans. Smart Grid, vol. 4, no. 1, pp. 321-331, March 2013.

[13] S. Gorshe, A. Raghavan, T. Starr and S. Galli, Power line communication, Wiley Telecom, 2014.

[14] H. Nosato et al, "A very low-cost low-frequency PLC system based on DS-CDMA for DC power lines," in Proc. 16th Int. Symp. Power Line Commun. Its Appl. (ISPLC), pp. 398-403, 2012.

[15] X. He, R. Wang, J. Wu, et al, "Nature of power electronics and integration of power conversion with communication for talkative power". Nat Commun, 2020, 11: 2479.

[16] J. Wu et al., "Power conversion and signal transmission integration method based on dual modulation of DCDC converters, " IEEE Trans. Ind. Electron., vol. 62, no. 2, pp. 1291-1300, Feb. 2015.

[17] R. Wang et al., "Direct sequence spread spectrum-based PWM strategy for harmonic reduction and communication, " IEEE Trans. Power Electron., vol. 32, no. 6, pp. 4455-4465, June 2017.

[18] J. Rodríguez, D. G. Lamar, D. G. Aller, P. F. Miaja and J. Sebastián, " Reproducing multi-carrier modulation schemes for visible light communication with the ripple modulation technique, " IEEE Trans. Ind. Electron., vol.67, no. 2, pp. 1532-1543, Feb. 2020.

[19] H. Choi and J. Jung, "Enhanced power line communication strategy for DC microgrids using switching frequency modulation of power converters," IEEE Trans. Power Electron., vol. 32, no. 6, pp. 4140-4144, June 2017.

[20] Y. Zhu et al., "Embedding power line communication in Photovoltaic optimizer by modulating data in power control loop", IEEE Trans. Ind. Electron., vol. 66, no. 5, pp. 3948-3958, May 2019.

[21] J. Du et al., "DC power-line communication based on power/signal dual modulation in phase shift full-bridge converters," IEEE Trans. Power Electron., vol. 32, no. 1, pp. 693-702, Jan. 2017.

[22] M. Angjelichinoski, Č. Stefanović, P. Popovski, H. Liu, P. C. Loh and F. Blaabjerg, "Multiuser communication through power talk in DC microgrids," IEEE Journal on Selected Areas in Communications, vol. 34 no. 7, pp. 2006-2021, July 2016.

[23] R. Chang and R. Gibby, "A theoretical study of performance of an orthogonal multiplexing data transmission scheme," IEEE Trans. Communication Technology, vol. 16, no. 4, pp. 529-540, Aug. 1968.

[24] G. Liu, T. Caldognetto, P. Mattavelli and P. Magnone, "Suppression of second-order harmonic current for droop-controlled distributed energy resource converters in DC microgrids," IEEE Trans. Ind. Electron., vol. 67, no. 1, pp. 358-368, Jan. 2020.

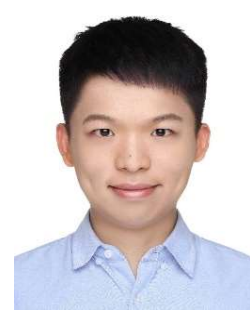

Ruoqi Zhang (S'18) was born in Fujian, China, in 1994. He received the B.S degree in electrical engineering from Zhejiang University, Hangzhou, China, in 2016. Currently, he is working toward the $\mathrm{Ph} . \mathrm{D}$. degree in the College of Electrical Engineering, Zhejiang University, Hangzhou, China.

From Sept. 2015 to June 2016, he was an intern at Silan Microelectronics Co. Ltd, Hangzhou, China. His current research interests include communication techniques applied in distributed power electronics

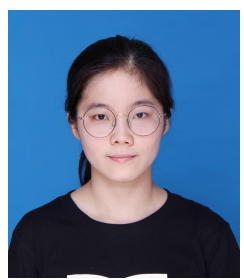

Yue Hui received the B.S degree in electrical engineering from Zhejiang University, Hangzhou, China, in 2020. Currently, she is working toward the M.S degree in the College of Electrical Engineering, Zhejiang University, Hangzhou, China. Her current research interests include distributed power electronics system.

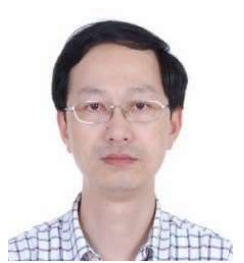

Jiande Wu (M'11) was born in Zhejiang, China, in 1973. He received the B.Sc., M.SC and Ph.D. degree from the College of Electrical Engineering, Zhejiang University, Hangzhou, China, in 1994, 1997 and 2012, respectively. Since 1997, he has been a faculty member at Zhejiang University, where he is currently an associate professor. From 2013 to 2014, he was an academic visitor at the University of Strathclyde, Glasgow, U.K. His research interests include power electronics control, distributed power electronics system and fieldbus communication.

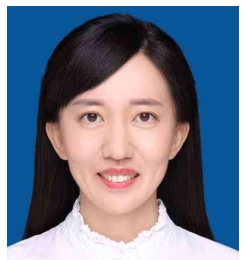

Ruichi Wang (S' 14--M' 19) received the B.Sc. and $\mathrm{Ph} . \mathrm{D}$ degree in electrical engineering from Zhejiang University, Hangzhou, China, in 2013 and 2019, respectively. Currently, she is working as Marie Skłodowska-Curie Actions - Individual Fellowships (MSCA-IF) in Loughborough University, UK. Her current research interests include information theory and communication technique applied in power electronics.

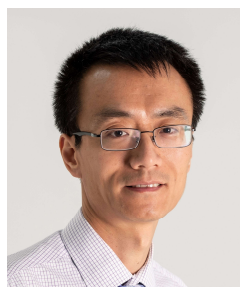

Zhengyu Lin (S'03-M'05-SM'10) received the B.Sc. and M.Sc. degrees from the College of Electrical Engineering, Zhejiang University, Hangzhou, China, in 1998 and 2001, respectively, and the Ph.D. degree from Herio-Watt University, Edinburgh, U.K., in 2005. He is currently a Senior Lecturer in the School of Mechanical, Electrical and Manufacturing Engineering, Loughborough University, Loughborough, UK.

His research interests include power electronics and its applications in renewable energy, energy storage, motor drives, microgrids, and multi-energy systems. He is currently holding an EPSRC UKRI Innovation Fellowship on DC microgrids.

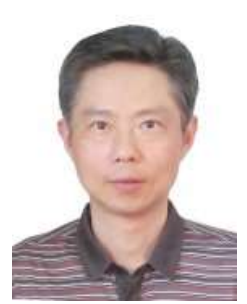

Xiangning He (M'95--SM'96--F'10) received the B.Sc. and M.Sc. degree from Nanjing University of Aeronautical and Astronautical, Nanjing, China, in 1982 and 1985, respectively, and Ph.D. degree from Zhejiang University, Hangzhou, China, in 1989.

In 1991, he obtained a Fellowship from the Royal Society of U.K., and conducted research in Heriot-Watt University, Edinburgh, U.K., as a Post-Doctoral Research Fellow for two years. In 1994, he joined Zhejiang University as an Associate Professor. Since 1996, he has been a Full Professor in the College of Electrical Engineering, Zhejiang University. His research interests are power electronics and their industrial applications.

Dr. He is a Fellow of The Institute of Electrical and Electronics Engineers (IEEE) and has been appointed as IEEE Distinguished Lecturer by the IEEE Power Electronics Society in 2011. He is also a Fellow of the Institution of Engineering and Technology (formerly IEE), U.K. 